\title{
Frequency-Domain Maximum-Likelihood Estimation of High-Voltage Pulse Transformer Model Parameters
}

\author{
Davide Aguglia, Member, IEEE, Philippe Viarouge, Member, IEEE, and Carlos de Almeida Martins, Member, IEEE
}

\begin{abstract}
This paper presents an offline frequency-domain nonlinear and stochastic identification method for equivalent model parameter estimation of high-voltage pulse transformers. Such kinds of transformers are widely used in the pulsed-power domain, and the difficulty in deriving pulsed-power converter optimal control strategies is directly linked to the accuracy of the equivalent circuit parameters. These components require models which take into account electric fields energies represented by stray capacitance in the equivalent circuit. These capacitive elements must be accurately identified, since they greatly influence the general converter performances. A nonlinear frequency-based identification method, based on maximum-likelihood estimation, is presented, and a sensitivity analysis of the best experimental test to be considered is carried out. The procedure takes into account magnetic saturation and skin effects occurring in the windings during the frequency tests. The presented method is validated by experimental identification of a $2-\mathrm{MW}-100-\mathrm{kV}$ pulse transformer.
\end{abstract}

Index Terms-High-voltage (HV) techniques, identification, maximum-likelihood (ML), optimization methods, pulse transformers.

\section{NOMENCLATURE}

$C_{1} \quad$ Primary distributed capacitance

$F$.

$C_{12}^{\prime}$ Primary-secondary capacitance (referred to primary) $F$.

$C_{2}^{\prime} \quad$ Secondary distributed capacitance

$F$.

Primary leakage inductance

$H$.

$L_{2}^{\prime} \quad$ Secondary leakage inductance (referred to primary) $H$.

$L_{m} \quad$ Magnetizing inductance

$R_{1} \quad$ Primary-winding resistance

$R_{2}^{\prime} \quad$ Secondary winding resistance (referred to primary)

$R_{f e} \quad$ Iron loss equivalent resistance

Manuscript received October 27, 2012; revised February 10, 2013; accepted March 21, 2013. Date of publication May 30, 2013; date of current version November 18, 2013. Paper 2012-EMC-517.R1, presented at the 2012 International Conference on Electrical Machines, Marseille, France, September 2-5, and approved for publication in the IEEE TRANSACTIONS ON INDUSTRY APPLICATIONS by the Electric Machines Committee of the IEEE Industry Applications Society.

D. Aguglia is with the European Organization for Nuclear Research (CERN), 1211 Geneva, Switzerland, and also with Laval University, Québec City, QC G1V 0A6, Canada (e-mail: davide.aguglia@cern.ch).

P. Viarouge is with the Laboratoire d'Électrotechnique, Électronique de Puissance et Commande Industrielle (LEEPCI), Department of Electrical Engineering, Laval University, Québec City, QC G1V 0A6, Canada (e-mail: philippe. viarouge@gel.ulaval.ca).

C. A. Martins is with the European Spallation Source, 22100 Lund, Sweden (e-mail: carlos.martins@esss.se).

Color versions of one or more of the figures in this paper are available online at http://ieeexplore.iee.org.

Digital Object Identifier 10.1109/TIA.2013.2265213

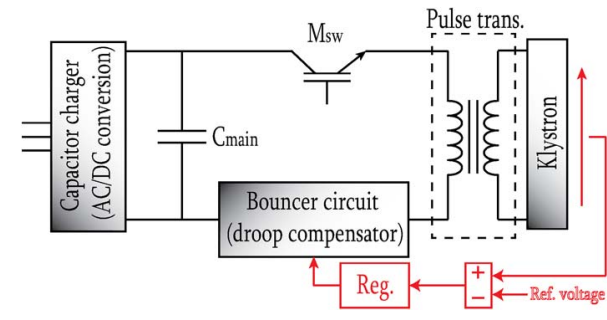

Fig. 1. Typical capacitor discharge-based klystron modulator topology.

$k \quad$ Transformer turns ratio

$X_{\text {ref }}$ Parameter value of the reference model.

\section{INTRODUCTION}

$\mathbf{H}$ IGH-VOLTAGE (HV) pulse transformers are widely used in the pulsed-power domain, such as radar applications, medicine, particle physics research, and food and material industry [1]. In these applications, such kinds of transformers are used to supply klystron tubes which produce high radio frequency (RF) power or RF antennas for plasma production. Often, this RF power is required for very short periods of time in a repetitive sequence. These pulsed-power converters present a typical topology consisting of a capacitor charger connected to a capacitor bank and a discharging converter producing the required output voltage pulse [2]. The output voltage pulse can be produced with different power converter topologies such as a direct switch topology [3], a solid-state Marx topology [4]-[6], or pulse-transformer-based topologies [7], [8]. The topology selection highly depends on the output voltage pulse shape and repetition rate. Pulse-transformer-based topologies are quite common due to their simplicity and reliability. Such kind of transformers can have slight geometrical differences: the monolithic-core "classical" transformer and the split-core or "matrix" transformer [9]. A typical topology of transformerbased klystron modulator is illustrated in Fig. 1, where an active bouncer circuit (switch mode power converter) is used for output voltage closed-loop regulation, which is used to compensate for the main capacitor $C_{\text {main }}$ voltage droop during the pulse.

A typical voltage shape to be produced with a so-called "long pulse" klystron modulator (power converter feeding klystrons) for particle accelerators (particularly linear accelerators) presents a flat top of a length between a few hundreds of microseconds and a few milliseconds, and a typical voltage range between 100 and $200 \mathrm{kV}$. 
The objective is to achieve a perfectly square-shaped voltage pulse, minimizing rise and fall times in order to minimize power losses in the load. For instance, a new accelerator under development is aiming for klystron modulators producing $150-\mathrm{kV}$ pulsed voltage with $140 \mu$ s of flat top, $3 \mu$ s of rise and fall times, and a pulse power of $23 \mathrm{MW}$ with a repetition rate of $50 \mathrm{~Hz}[10]$.

For the new generation of klystron modulators based on pulse transformers, the accurate knowledge of every equivalent circuit's parameter is critical for achieving higher and higher performances [11], [12]. Several works have been presented in the literature regarding identification of power transformers, such as those in [13] and [14], where recursive methods are employed on frequency response data, or those in [15] and [16], where artificial neural-network-based methods are used. For control purposes, the parametric identification of any accurate mathematical model relating the input to the output of a system could be sufficient; however, for the transformer designer, a model with parameters representing its physical behavior is required. To experimentally validate the design models in [17], this paper presents an identification method of the classical high-frequency (HF) equivalent circuit of a power transformer. The model takes into account distributed and interwinding lumped capacitances. An important contribution of this work is the consideration of the winding skin effect and magnetic saturation of the transformer core which occurs during frequencybased identification tests.

This paper presents the general method along with the necessary models for different test conditions and methods for taking into account skin and magnetic saturation effects. A method for the selection of the most suitable frequency tests is proposed, as well as an experimental identification of a $2-\mathrm{MW}-100-\mathrm{kV}$ pulse transformer.

\section{IDENTIFICATION METHOD AND REQUIRED TRANSFORMER MODELS}

\section{A. Identification Method and General Algorithm}

To estimate the parameters of the equivalent circuit model of the pulse transformer, a numerical optimization method is used for minimizing a given objective function by iteratively changing the model parameters to be estimated. The general optimization problem can be stated as in

$$
\begin{aligned}
& \min V(\theta) \\
& \text { subject to }\left\{\begin{array}{l}
\theta-\theta_{\min } \geq 0 \\
\theta_{\max }-\theta \geq 0
\end{array}\right.
\end{aligned}
$$

where $V(\theta)$ is the objective function, or criterion, to be minimized and $\theta$ is the vector of parameters to be identified. The general algorithm of the numerical optimization method is illustrated in Fig. 2. The considered equivalent circuit of the $H V$ pulse transformer is shown in Fig. 3, leading to the parameter vector to be estimated in

$$
\theta=\left[C_{1} C_{12}^{\prime} C_{2}^{\prime} R_{1} R_{2}^{\prime} R_{f e} L_{1} L_{m} L_{2}^{\prime}\right]
$$

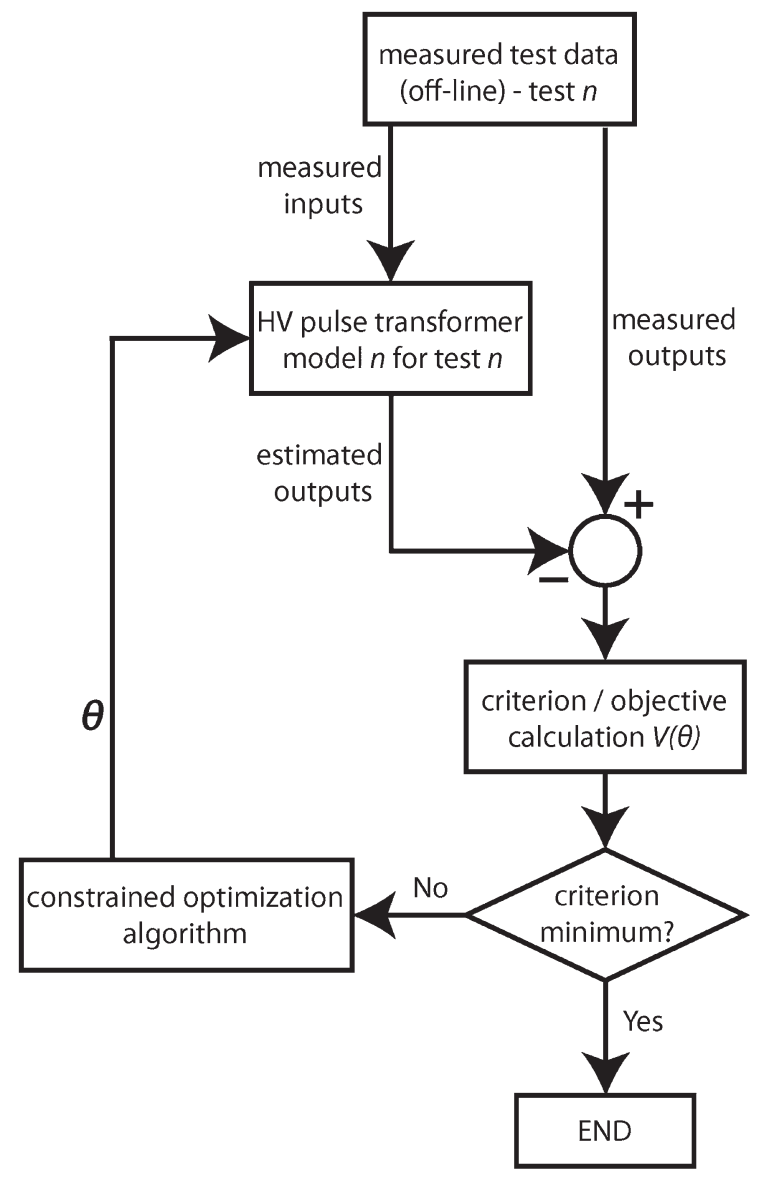

Fig. 2. General algorithm of the nonlinear identification process.

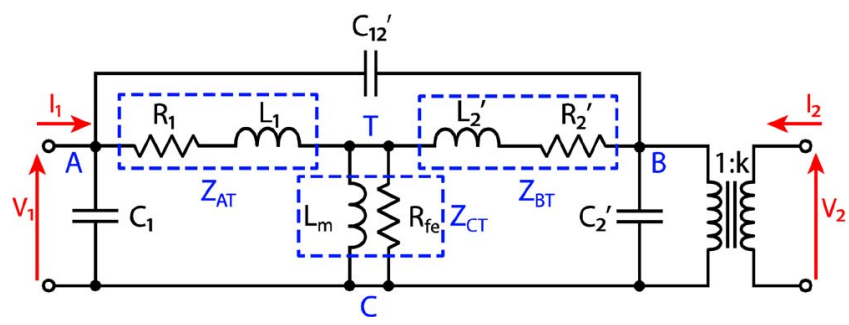

Fig. 3. Primary-referred HV pulse transformer equivalent circuit.

The transformer ratio $k$ is supposed to be known, and all parameters are referred to primary.

\section{B. Transformer Models for Frequency-Domain Identification}

Since the identification tests considered in this paper are in the frequency domain, transfer functions must be derived from the equivalent circuit shown in Fig. 3. This circuit includes the classical low-frequency magnetic transformer model, combined with the electrostatic one, which is represented by the primaryand secondary-winding distributed capacitances $C_{1}$ and $C_{2}^{\prime}$ and the primary-secondary interwinding capacitance $C_{12}^{\prime}$. This is the standard circuit used for representing the dynamics of $\mathrm{HV}$ pulse transformers [18]. In normal operation of this kind of transformers, the lumped parameter model is accurate enough to represent the behavior of its dynamics (rise/fall times and converter switching harmonic attenuation). In the experimental 


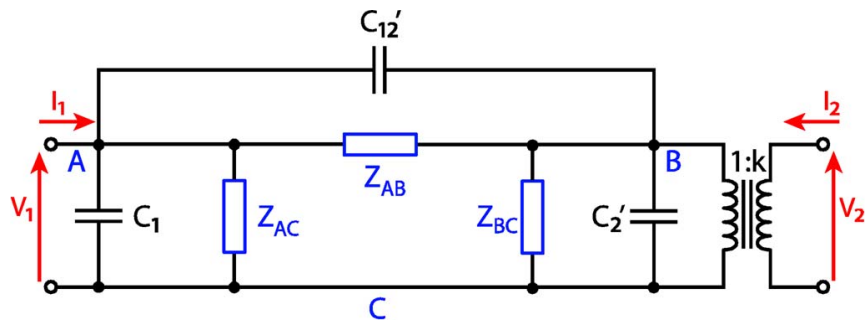

Fig. 4. Primary-referred HV pulse transformer equivalent circuit with Kennelly transformation on resistive and magnetic components.

validation in Section $\mathrm{V}$, the accuracy of this model is verified as well.

Modeling a distributed capacitance with a single lumped parameter is not as precise as considering a model with several distributed $R L C$ cells [16] (in [19], a 31-order model is used), particularly if very fast transients must be evaluated (such as arc events). However, this work has been also carried out with the objective of validating and verifying electromagnetic design processes, which include finite-element analyses (FEAs) and are based on lumped parameter models for efficient optimization. This is why the relatively simplified lumped parameter equivalent circuit in Fig. 3 is used.

An important aspect in the parametric identification process consists in the selection of the best test which excites the highest number of modes of the system to be identified. There are five possible frequency response tests which can be performed from the transformer terminals:

$n_{1} \quad V_{2} / V_{1}=f$ (freq) at no load;

$n_{2} \quad V_{1} / I_{1}=f($ freq $)$, characteristic impedance at no load;

$n_{3} \quad V_{1} / I_{1}=f($ freq), characteristic impedance with secondary winding in short circuit;

$n_{4} \quad V_{2} / I_{2}=f$ (freq), characteristic impedance at no load;

$n_{5} \quad V_{2} / I_{2}=f($ freq $)$, characteristic impedance with primary winding in short circuit.

Each of these tests could be an excellent candidate for the identification process. It is not trivial to derive the best test to retain; therefore, every test is analyzed from different points of view in what follows.

To derive the transfer function model representative of each one of the five tests $\left(n_{1}\right.$ to $\left.n_{5}\right)$, from the equivalent circuit in Fig. 3, one can perform a Kennelly transform (wye to delta) [20] of the magnetic elements in order to simplify the analytical equations. From the circuit in Fig. 3, it is then possible to obtain the new circuit in Fig. 4, which is much simpler to analyze for deriving the five different transfer functions. After mathematical manipulation, the transfer function forms for each of the five tests are as follows.

1) For $n_{1}$,

$$
\frac{V_{2}}{V_{1}}=k \frac{A_{1} s^{4}+B_{1} s^{3}+C_{1} s^{2}+D_{1} s}{E_{1} s^{4}+F_{1} s^{3}+G_{1} s^{2}+H_{1} s+I} .
$$

2) For $n_{2}$,

$$
\frac{V_{1}}{I_{1}}=\frac{A_{2} s^{4}+B_{2} s^{3}+C_{2} s^{2}+D_{2} s+E_{2}}{F_{2} s^{5}+G_{2} s^{4}+H_{2} s^{3}+I_{2} s^{2}+L_{2} s+M_{2}} .
$$

TABLE I

Typical Equivalent Circuit PARAMETERS OF A 2-MW-100-kV 800- $\mu$ s PUlSE TRANSFORMER

\begin{tabular}{ccc}
\hline \hline parameter & value & unit \\
\hline \hline $1: \mathrm{k}$ & $1: 10$ & - \\
$\mathrm{R}_{1 \_ \text {ini }}$ & 1 & $\Omega$ \\
$\mathrm{R}_{2}$ ini & 1 & $\Omega$ \\
$\mathrm{R}_{\text {fe_ini }}$ & 10 & $k \Omega$ \\
$\mathrm{L}_{1 \_ \text {ini }}$, & 100 & $\mu H$ \\
$\mathrm{~L}_{2 \_ \text {ini }}$ & 100 & $\mu H$ \\
$\mathrm{~L}_{\mathrm{m} \_ \text {ini }}$ & 0.6 & $H$ \\
$\mathrm{C}_{1}$ _ini & 10 & $p F$ \\
$\mathrm{C}_{2 \_ \text {ini }}$, & 10 & $\mu F$ \\
$\mathrm{C}_{12 \text { ini }}$ & 1 & $n F$ \\
\hline \hline
\end{tabular}

3) For $n_{3}$,

$$
\frac{V_{1}}{I_{1}}=\frac{A_{3} s^{3}+B_{3} s^{2}+C_{3} s+D_{3}}{E_{3} s^{4}+F_{3} s^{3}+G_{3} s^{2}+H_{3} s+I_{3}} .
$$

4) For $n_{4}$,

$$
\frac{V_{2}}{I_{2}}=\frac{A_{4} s^{4}+B_{4} s^{3}+C_{4} s^{2}+D_{4} s+E_{4}}{F_{4} s^{5}+G_{4} s^{4}+H_{4} s^{3}+I_{4} s^{2}+L_{4} s+M_{4}} .
$$

5) For $n_{5}$,

$$
\frac{V_{2}}{I_{2}}=\frac{A_{5} s^{3}+B_{5} s^{2}+C_{5} s+D_{5}}{E_{5} s^{4}+F_{5} s^{3}+G_{5} s^{2}+H_{5} s+I_{5}} .
$$

Coefficients for tests $n_{1}$ and $n_{2}$ are presented in the Appendix in MatLab "*.m" file format. Looking at expressions (3)-(7), one can notice that test $n_{2}$ and $n_{4}$ (primary and secondary impedances at no load) are the ones which present the highest number of modes. The modes of each test are excited at frequencies which depend on transformer geometry and dimensions. To evaluate the frequency behavior of each test, a typical $H V$ pulse transformer equivalent circuit has been considered. Table I shows the typical rough parameter values of a megawattrange pulse transformer (flat top in the millisecond range).

These parameters will be used in Section $\mathrm{V}$ as initial conditions for the experimental identification. These hypothetic transformer parameters have been considered for the evaluation of the five tests described by the transfer function in (3)-(7). The simulated frequency responses of each test are presented in Fig. 5.

Notice how the higher order transfer function for $n_{2}$ test in (4) is translated into resonance and antiresonance peaks in Fig. 5. The same order is achieved in test $n_{4}$ expressed in (6); however, all resonances and antiresonances are appearing in a much higher frequency region (out of scale in Fig. 5). When considering practical experimental setups, one has to bear in mind the possible limitations of measuring instrumentation. Looking at the results from Fig. 5, it has been decided that test $n_{3}$ (primary impedance with secondary in short circuit) would not be considered for identification purposes. Indeed, the available current measuring instrumentation keeps a very good precision in amplitude and phase up to $1 \mathrm{MHz}$.

One possible solution to cope with these practical issues would be to alter the behavior of the system by adding external components. For instance, one can imagine adding a capacitor, an inductor, or a resistor in series to the variable frequency 

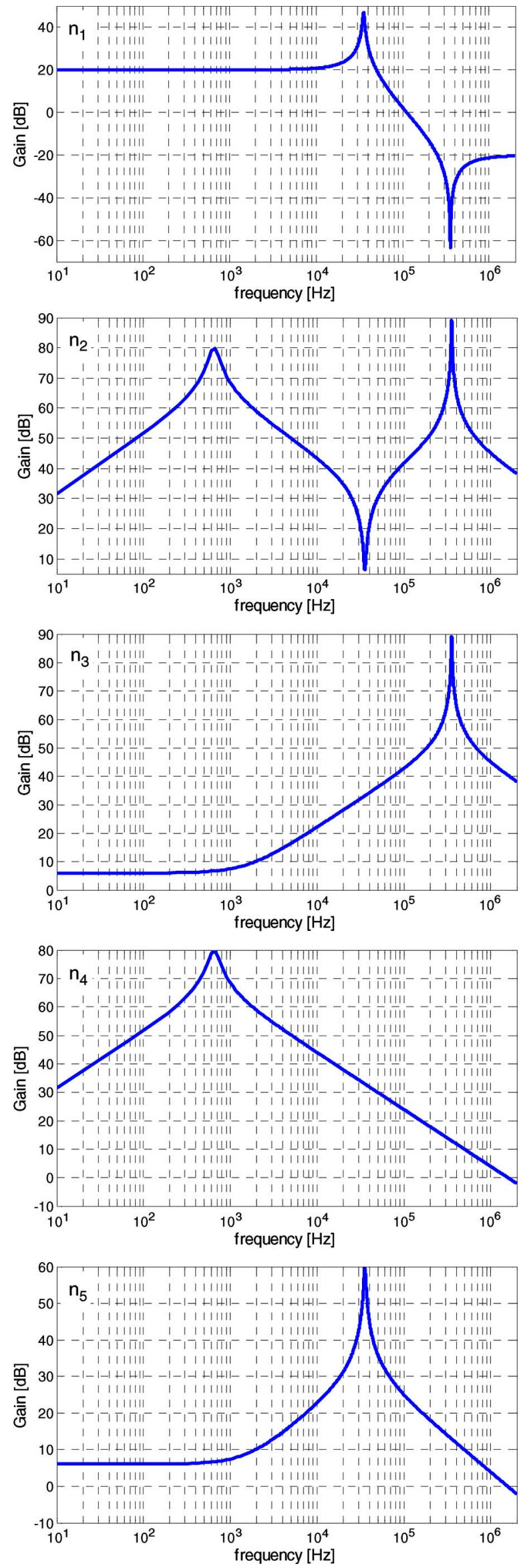

Fig. 5. Frequency response of transfer function in (3)-(7) corresponding to tests $n_{1}$ to $n_{5}$, for the transformer parameters presented in Table I. supply or in parallel to a winding in order to modify the position of the equivalent model poles or zeros. In this case, an accurate frequency identification of the added elements must be carried out separately.

By adopting this method, tests which may seem not interesting or not experimentally viable (as the $n_{3}$ test in this case) could become interesting. For the presented work, this alternative has not been further developed or tested for the sake of minimizing the experimental procedure complexity and time.

\section{Considering Magnetic Saturation and Skin Effects}

The problem of frequency-based identification methods is given by the severe change of operating conditions of the transformer (wide-range frequency sweeps). For instance, if no attention is paid to the saturation level of the iron core, the identified parameters will be heavily biased.

Furthermore, skin effect drastically changes the winding resistances during frequency sweeps. These two phenomena shall be taken into account for an accurate identification process, which means that the models in (3)-(7) become nonlinear. These kinds of transformers are designed for very short pulses, meaning that the iron core cross section is relatively small. As such, during experimental measurements in the low-frequency region, one can reach deep saturation levels even if the supplying voltage is very low.

A way of getting rid of magnetic saturation effect during experimental measurement consists in performing a frequency sweep respecting a constant voltage/frequency law. Depending on available equipment, this could be time consuming if no automated processes can be foreseen. Saturation and skin effects can be considered by extending the model order as in [21][24], where additional parameters, describing the nonlinearity of these phenomena, can be identified together with the rest of the transformer parameters. However, the authors of this paper decided to avoid such an approach and tried to keep unchanged the order of the equivalent circuit in Fig. 3 for parameter physical description purposes.

A way of considering saturation phenomena without modifying the circuit in Fig. 3 consists in adapting the nonsaturated magnetizing inductance value, at each operating point of the transformer, by multiplying it with a predetermined coefficient $k_{\text {sat }}(B)$ [25]. In this case, the magnetizing inductance is computed as follows:

$$
L_{m}=k_{\mathrm{sat}}(B) L_{m}^{0}
$$

with $L_{m}^{0}$ as the nonsaturated magnetizing inductance value which becomes the identification variable, instead of $L_{m}$ in (2). $k_{\text {sat }}(B)$ is derived from a priori measurements and depends on the operating point of the transformer.

Knowing the winding number of turns and the magnetic core cross section (easily obtainable in this oil-insulated transformers), it is possible to measure the $B-H$ characteristic of the magnetic material (if not already known from data sheets). $K_{\text {sat }}(B)$ can be derived as the ratio between the saturated and nonsaturated magnetic permeabilities as in (9). Fig. 6 illustrates the principle for obtaining the saturation factor, which can 
(a)

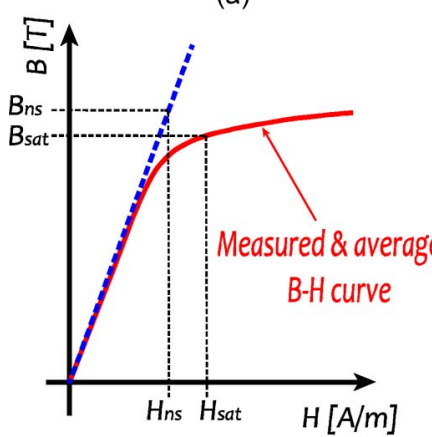

(b)

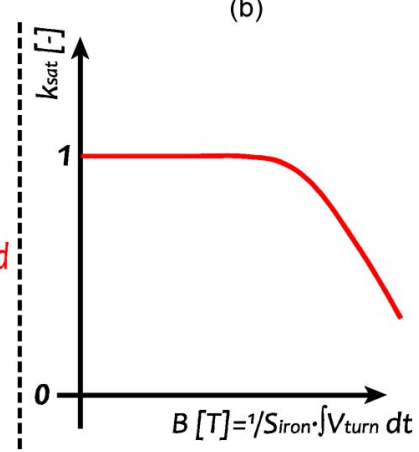

Fig. 6. Method for measuring the saturation factor $k_{\text {sat }}$.

be a direct function of the measured voltage input during the identification process.

For the identification program automation, the measured $k_{\text {sat }}$ coefficient can be fitted with a polynomial function or with a simple two-parameter ( $a$ and $b$ ) function as in (10) [26]

$$
\begin{aligned}
& k_{\mathrm{sat}}(B)=\frac{\mu_{\mathrm{sat}}}{\mu_{n s}}=\frac{B_{\mathrm{sat}} \cdot H_{n s}}{B_{n s} \cdot H_{\mathrm{sat}}} \\
& k_{\mathrm{sat}}(B)=\frac{1}{1+a \cdot B^{b}} .
\end{aligned}
$$

In such an approach, the optimization procedure has the objective of identifying a constant value $L_{m}^{0}$, which is irrespective from the saturation effect. Adopting this method, the model has been linearized by a measurement of the magnetic saturation nonlinearity. This saturation model has already been successfully used for electrical machine identification [27].

Skin effect may have a big impact in frequency-domain identification. Indeed, the lower voltage winding can present a copper cross section such that skin effects are noticeable starting from a few kilohertz. These step-up pulse transformers present a primary (low-voltage winding) made of a rectangular conductor (kiloampere range). The secondary winding is commonly made of a very tiny cylindrical conductor, and skin effects can be neglected up to $1 \mathrm{MHz}$. For cylindrical conductors, one can take into account skin effect including resistance adaptation factors analytically derived from Maxwell equation solutions based on Bessel functions [28]. For rectangular conductors, the resistance correction factor $k_{r}$ can be derived from the Poynting vector formulation [29]. This coefficient can be expressed as in

$$
k_{r}(f)=\frac{R_{\mathrm{ac}}}{R_{\mathrm{dc}}}=\xi \frac{\sinh (2 \xi)+\sin (2 \xi)}{\cosh (2 \xi)-\cos (2 \xi)}
$$

with

$$
\xi=\alpha h \quad \alpha=\sqrt{\frac{\pi \mu_{0} f}{\rho_{\mathrm{Cu}}}}
$$

where $\alpha$ is the inverse of the skin depth, $\rho_{\mathrm{Cu}}$ is the copper resistivity, and $h$ is the height of the rectangular conductors (the width is simplified but considered in the calculation of the dc resistance $R_{\mathrm{dc}}$ ).

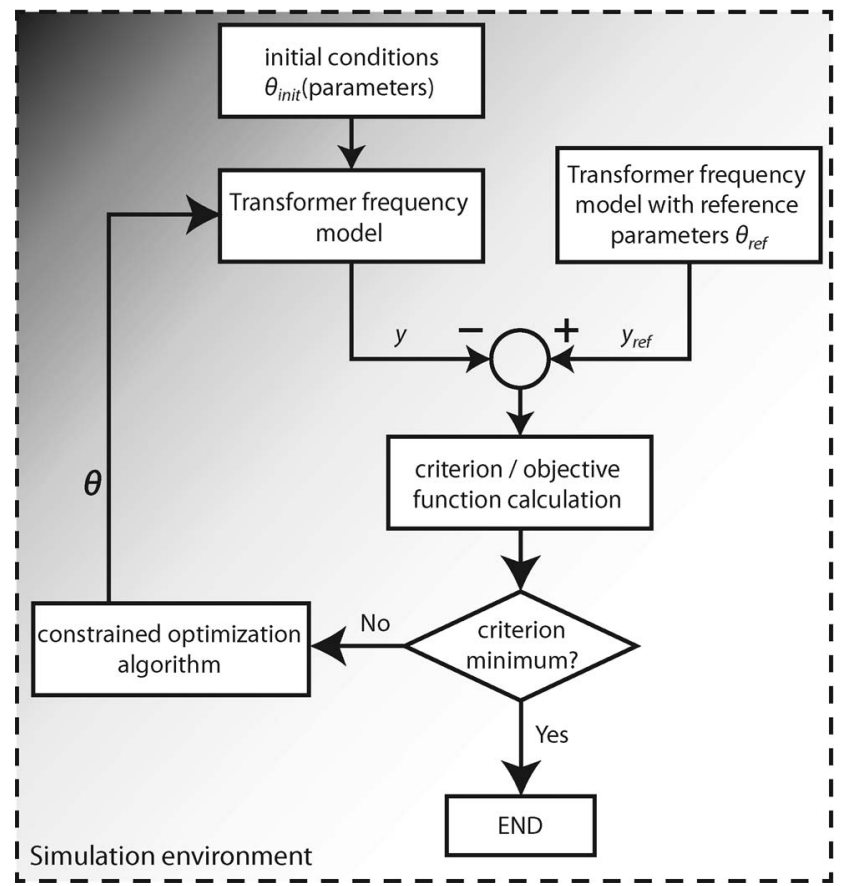

Fig. 7. Algorithm for nonlinear identification convergence analysis.

It is demonstrated in [30] that, if the conductor cross section is square, the skin effect is identical to the one occurring in a cylindrical conductor with a diameter that is equal to the side of the squared one (if the primary conductor appends to be cylindrical). Considering the skin effect model in (11) and (12), one linearizes the identification process by knowing the material and geometry of winding conductors.

As previously mentioned, the transfer function models presented in (3)-(7) become nonlinear if magnetic saturation and skin effects are considered. For each frequency under evaluation, coefficients $k_{\text {sat }}$ and $k_{r}$ must be computed and updated in the transfer functions (3)-(7) in order to linearize the identification process.

\section{Identification Test SElection}

A typical problem arising when using nonlinear identification methods involving several variables consists in the verification of the presence of a global minimum in the objective function. Among several parameters influencing the convergence of such methods are a good limitation of the solution space owing to variable constraints and a good selection of initial variable conditions. Furthermore, the test nature and the form of the objective function greatly influence the optimization convergence. In what follows, a method for determining the best frequency identification test for HV pulse transformers is presented. The methodology is described in Fig. 7 and consists in emulating experimental measurements by simulation of a known model, with $\theta_{\text {ref }}$ as reference parameter vector, on the selected four frequencies tests $\left(n_{1}, n_{2}, n_{4}\right.$, and $\left.n_{5}\right)$. These emulated test results are then used to reidentify a model starting from several sets of parameter initial conditions. This procedure allows determining the robustness of the nonlinear identification method by verifying that the estimated parameters 
TABLE II

Identification Method Convergence Sensitivity AnALysis

\begin{tabular}{|c|c|c|c|c|c|c|c|}
\hline $\begin{array}{l}\text { Init. } \\
\text { Cond }\end{array}$ & $\begin{array}{c}\text { Emulated } \\
\text { test }\end{array}$ & $\frac{R_{1}}{R_{1 \_r e f}}$ & $\frac{R_{2}}{R_{2 \_ \text {ref }}}$ & $\frac{L_{1}}{L_{1 \_ \text {ref }}}$ & $\frac{L_{2}}{L_{2 \_r e f}}$ & $\frac{L_{m}}{L_{m_{\text {_r }} r f f}}$ & $\frac{C_{2}^{\prime}}{C_{2 \_r e f}^{\prime}}$ \\
\hline \multirow{5}{*}{$\begin{array}{l}i=1 \\
j=1\end{array}$} & $\sum \mathrm{n}_{1,2,4,5}$ & 1 & 1 & 1 & 1 & 1 & 1 \\
\hline & $\mathrm{n}_{1}$ & 0.82 & 1.05 & 0.94 & 0.94 & 0.82 & 1.07 \\
\hline & $\mathrm{n}_{2}$ & 1 & 1 & 1 & 1 & 1 & 1 \\
\hline & $n_{4}$ & 1.39 & 1 & 1 & 1 & 1 & 1 \\
\hline & $\mathrm{n}_{5}$ & 1.09 & 0.90 & 1 & 1 & 1.13 & 0.99 \\
\hline \multirow{5}{*}{$\begin{array}{l}i=-1 \\
j=-1\end{array}$} & $\sum \mathrm{n}_{1,2,4,5}$ & 1 & 1 & 1 & 1 & 1 & 1 \\
\hline & $\mathrm{n}_{1}$ & 0.33 & 0.56 & 0.44 & 0.43 & 4.53 & 2.28 \\
\hline & $\mathrm{n}_{2}$ & 1 & 1 & 1 & 1 & 1 & 1 \\
\hline & $\mathrm{n}_{4}$ & 0.60 & 0.01 & 0.6 & 0.6 & 1 & 1 \\
\hline & $\mathrm{n}_{5}$ & 0.01 & 1.99 & 1 & 1 & 14 & 1.01 \\
\hline \multirow{5}{*}{$\begin{array}{l}i=1 \\
j=-1\end{array}$} & $\Sigma \mathrm{n}_{1,2,4,5}$ & 1 & 1 & 1 & 1 & 1 & 1 \\
\hline & $\mathrm{n}_{1}$ & 0.56 & 0.95 & 0.91 & 0.58 & 0.57 & 1.34 \\
\hline & $\mathrm{n}_{2}$ & 1 & 1 & 1 & 1 & 1 & 1 \\
\hline & $\mathrm{n}_{4}$ & 1.4 & 1 & 1 & 0.6 & 1 & 1 \\
\hline & $\mathrm{n}_{5}$ & 1.13 & 0.86 & 1 & 1 & 1.14 & 0.98 \\
\hline \multirow{5}{*}{$\begin{array}{l}i=-1 \\
j=1\end{array}$} & $\sum \mathrm{n}_{1,2,4.5}$ & 1 & 1 & 1 & 1 & 1 & 1 \\
\hline & $\mathrm{n}_{1}$ & 0.36 & 0.61 & 0.29 & 0.69 & 6.4 & 2 \\
\hline & $\mathrm{n}_{2}$ & 1 & 1 & 1 & 1 & 1 & 1 \\
\hline & $\mathrm{n}_{4}$ & 0.58 & 1 & 0.6 & 1 & 1 & 1 \\
\hline & $\mathrm{n}_{5}$ & 1.17 & 0.83 & 1 & 1 & 14.3 & 1 \\
\hline
\end{tabular}

$\theta$ converge to the reference one $\theta_{\text {ref }}$, for different initial conditions and frequency test nature. It is reasonable to suppose that a preliminary estimation of each parameter, following classical test procedures (no-load and short-circuit tests), leads to a maximal $50 \%$ of error on each parameter. This study evaluates four sets of initial conditions $\theta_{\text {init }}$ which consider an overestimation and an underestimation of initial values, as shown in

$$
\theta_{\text {init }}=0.5 \theta_{\text {ref }}[i j i j i j i j i]^{\mathrm{T}}
$$

where $i$ and $j$ can adopt the values of 1 or -1 . For instance, if $i=1$ and $j=-1$, the initial conditions are

$$
\begin{gathered}
\theta_{\text {init }}=0.5\left[C_{1 \_ \text {ref }}-C_{12 \_ \text {ref }}^{\prime}-C_{2 \_ \text {ref }}^{\prime}-R_{1 \_ \text {ref }} \cdots R_{2 \_ \text {ref }}^{\prime}\right. \\
\left.-R_{f e \_r e f} L_{1 \_ \text {ref }}-L_{m \_r e f} L_{2 \_ \text {ref }}^{\prime}\right] .
\end{gathered}
$$

In (14), the subscript "ref" refers to a parameter included in the reference parameter vector $\theta_{\text {ref }}$ (Fig. 7), which has been set with values in Table I. Results of this robustness, or convergence, analysis of the identification method are presented in Table II. A total of 20 identification processes have been carried out considering four different initial conditions (sets of $i-j$ values) and five-different-test nature. Having, for this simulated analysis, noise-free measurements, a classical least square objective function has been considered for minimization

$$
V_{L S}(\theta)=\sum_{k=1}^{n_{f}}\left(y_{\text {ref }}(k)-y(k)\right)^{2}=\sum_{k=1}^{n_{f}}(\varepsilon(k))^{2}
$$

where $f$ is the test frequency, $y$ is the gain of the transfer function corresponding to the test $\left(n_{1}, \ldots, n_{5}\right), n_{f}$ is the number of tested frequencies during the test sweep, and $\varepsilon$ is the residual vector. In Table II, $\Sigma n_{1,2,4,5}$ represents the sum of the four objective functions associated to the four tests $\left(n_{1}, n_{2}, n_{4}\right.$, and $\left.n_{5}\right)$. In this case, all four tests were consid- ered during the identification process, and the objective function was composed of the sum of the objective functions (15) of each test. However, in practice, performing all the tests at the same time for the identification process could be time consuming.

Table II shows the ratios between each identified parameter and its reference parameter. MatLab function fmincon has been used as optimization algorithm, and no variable constraints were used for this exercise.

In some cases, very high identification errors have been obtained, such as in $n_{5}$, where $L_{m} / L_{m \_ \text {ref }}=14.3$ or $R_{1} /$ $R_{1 \text { ref }}=0.01$. This is an indicator of the presence of local minima in the objective function; however, it is also a consequence of having no constraints on variables. Constraints have been deliberately omitted to identify the most "healthy" objective function, even when one does not know how to set constraint values and initial conditions. Notice that test $n_{1},\left(V_{2} / V_{1}\right)$, presents the worst results. As expected, if one considers all four tests and sums up the contribution of each one, the result is excellent. Excellent results are achieved with test $n_{2}$ alone as well, demonstrating that the no-load primary impedance test is the most suitable for the presented parametric identification method.

Furthermore, a no-load test is very easy to set, since no short circuit with special HV connectors is required (in contrast with tests $n_{3}$ and $n_{5}$ ). Another conclusion from this analysis is that the identification method, considering the no-load primary impedance test, easily converges to the same result for any initial condition. This analysis has been automatically repeated for a wide range of sets of reference parameters $\theta_{\text {ref }}$, and results are consistently indicating that $n_{2}$ test is by far the best one.

\section{ML PARAMETER IDENTIFICATION}

Experimental measurements involve noise, and its effect can have a great impact on the final estimated parameters, which tend to be biased, as well as on the convergence capability of the optimization process. In electrical machine parameter identification, maximum-likelihood (ML) estimation has been proven to be an excellent stochastic identification method for improving precision and convergence efficiency [23], [31][34]. If the likelihood function, in the form as in (16), is maximized, then the residuals $\left(\varepsilon=y_{\text {ref }}-y\right)$ tend to a withe and Gaussian noise sequence [35]

$$
L(\theta)=\sum_{k=1}^{n_{f}}\left[\frac{1}{\sqrt{2 \pi \operatorname{det}(R(\theta))}} \exp \left(-\frac{1}{2} \varepsilon(k)^{\mathrm{T}} R(\theta)^{-1} \epsilon(k)\right)\right]
$$

where $R(\theta)$ denotes the sample covariance matrix (17) of the residual vector $\varepsilon(k)$. Maximizing (16) is equivalent to minimizing its negative logarithm (18), which is more convenient for optimization purposes [35]. In (18), unnecessary constants from (16) have been ignored

$$
R(\theta)=\frac{1}{n_{f}} \operatorname{cov}\left(\varepsilon(k) \cdot \varepsilon(k)^{\mathrm{T}}\right)
$$




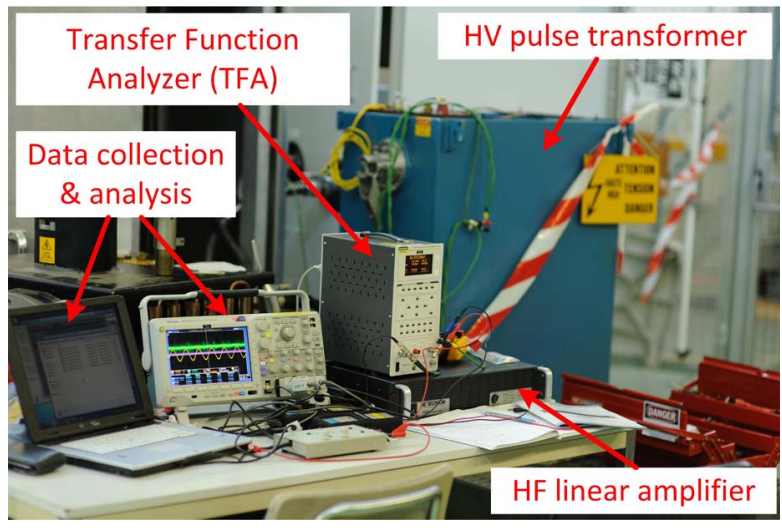

Fig. 8. View of the identification test experimental setup.

$$
\begin{aligned}
V_{\mathrm{ML}}(\theta)= & \frac{1}{2} \sum_{k=1}^{n_{f}}\left(\varepsilon(k)^{\mathrm{T}} R(\theta)^{-1} \varepsilon(k)\right) \\
& +\frac{1}{2} \mathcal{N} \ln (\operatorname{det}(R(k, \theta))) .
\end{aligned}
$$

For each optimization step of the identification method illustrated in Fig. 2, the covariance matrix $R(\theta)$ is recalculated, leading to an iterative reweighting of the objective function (18). The identification process can be summarized in the three following steps [32].

1) Fix $R(\theta)=I$ (where $I$ is the $n_{f}$ by $n_{f}$ identity matrix), and minimize (18) with respect to $\theta$. This corresponds to the objective function in (15).

2) Compute $R(\theta)$ from (17) using the residuals $\varepsilon$ from step 1).

3) Calculate the new objective function (18) with $R(\theta)$ from step 2), and minimize with respect to $\theta$.

Steps 2) and 3) are iteratively repeated by the optimization algorithm until convergence.

\section{EXPERIMENTAL ML IDENTIFICATION OF A 2-MW-100-kV PULSE TRANSFORMER}

\section{A. Experimental Setup}

A view of the experimental setup for the identification of a 2-MW-100-kV-800- $\mu$ s pulse transformer is shown in Fig. 8.

A high-precision transfer function analyzer (TFA) is used to perform the frequency sweep required for each test and to measure the considered input/output signals for the derivation of the gain-frequency characteristic. Fig. 9 illustrates the winding configuration of the transformer under test. The primary windings are made of a rectangular conductor to form a singlelayer coil. The two coils p1 and p2 are connected in parallel to form the primary winding. The secondary winding is composed of two coils connected in series. Notice that the distance from primary to secondary windings is not the same in the two legs of the transformer. The reason is to respect the minimum insulating distances (which must be higher for the higher voltage secondary coil s2), in order to minimize the total leakage inductance (lower rise time). The transformer is immerged in an oil tank, and the magnetic core material is made of a thin strip of silicon-iron material (to reduce magnetic losses and to (a)
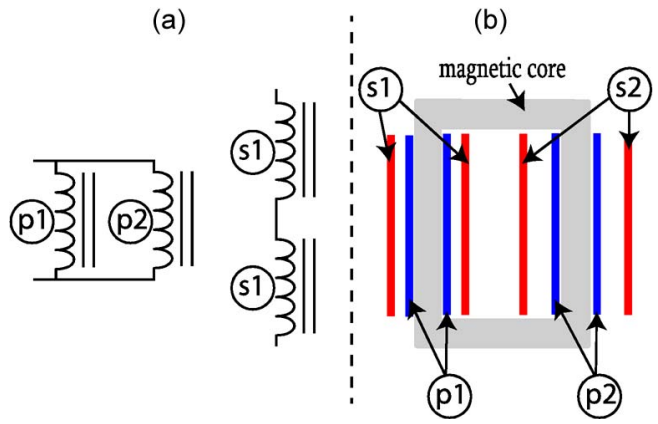

Fig. 9. Winding configuration of the transformer under test.
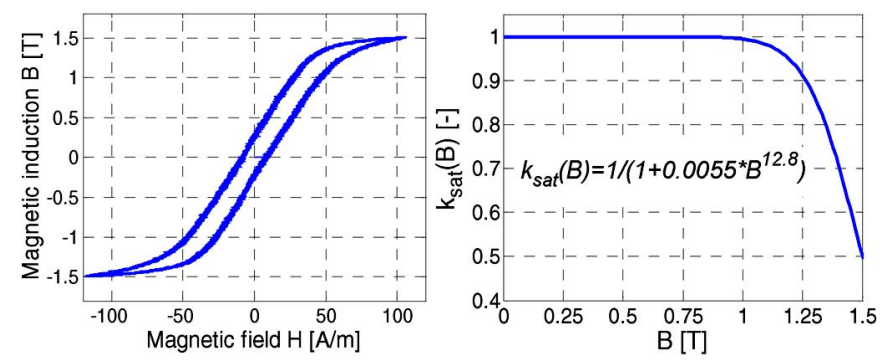

Fig. 10. (Left) Measured $B-H$ characteristic of the $H V$ pulse transformer magnetic core under sinusoidal excitation (4-Hz test). (Right) Corresponding saturation factor $k_{\text {sat }}$.

reduce the eddy-current dynamics for a faster voltage rise time). For current measurements, a high-precision/high-bandwidth $(1 \mathrm{MHz})$ shunt current transducer is used.

Voltages are directly measured by the TFA, which includes the feature of dynamically adapting the range of measurements in order to keep the maximum measuring precision during the frequency sweep. A 1-kW HF (1 MHz) linear amplifier is driven by the TFA and supplies the transformer (voltage source). This power amplifier is needed to supply the required magnetizing energy. The tests are performed considering a magnetic flux level corresponding to approximately two-thirds of the nominal value. The magnetic material has been characterized to measure the saturation coefficient as shown in Fig. 10. Skin effect in the primary winding has been considered by defining the coefficient $k_{r}$ from geometrical dimensions measured on the conductor.

\section{B. Experimental Results and Cross-Validation Tests}

The result of the identification considering test $n_{2}$ is presented in Fig. 11. Experimental data consist of 190 measured points. Fig. 11 shows the differences occurring when neglecting or considering magnetic saturation and skin effects. These results prove the need of taking into account these effects. Final parameters are presented in Table III. For validation purposes, it is important to cross-check the obtained result by comparing the obtained model on a different test. The performed additional test concerns the voltage transfer function $V_{2} / V_{1}$, which is representative for the pulse shape in time domain. Results are shown in Fig. 12.

The good agreements with experimental results prove the efficiency of the identification method. However, looking closer to the results, one can notice resonances in the nearby of 


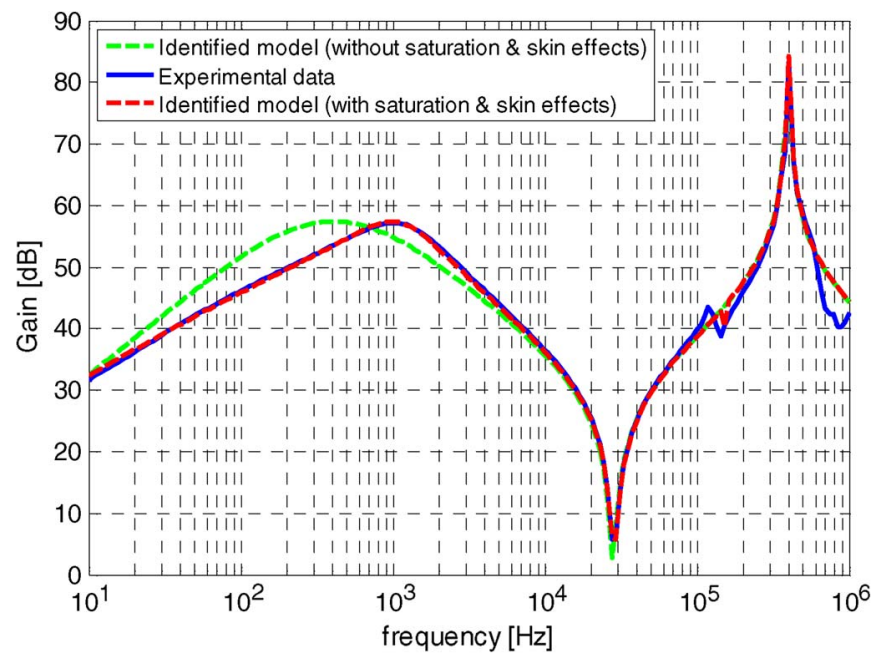

Fig. 11. ML identification result for the no-load primary impedance test $n_{2}$.

TABLE III

EXPERIMENTAL IDENTIFICATION RESULTS

\begin{tabular}{ccc}
\hline \hline parameter & value & unit \\
\hline \hline $\mathrm{R}_{1}$, & 0.21 & $\Omega$ \\
$\mathrm{R}_{2}$, & 0.49 & $\Omega$ \\
$\mathrm{R}_{\mathrm{fe}}$ & 460 & $\Omega$ \\
$\mathrm{L}_{1}$, & 68 & $\mu H$ \\
$\mathrm{~L}_{2}$ & 102 & $\mu H$ \\
$\mathrm{~L}_{\mathrm{m}}$ & 0.48 & $H$ \\
$\mathrm{C}_{1}$ & 1.9 & $p F$ \\
$\mathrm{C}_{2}$, & 0.27 & $\mu F$ \\
$\mathrm{C}_{12}$, & 1.45 & $n F$ \\
\hline \hline
\end{tabular}

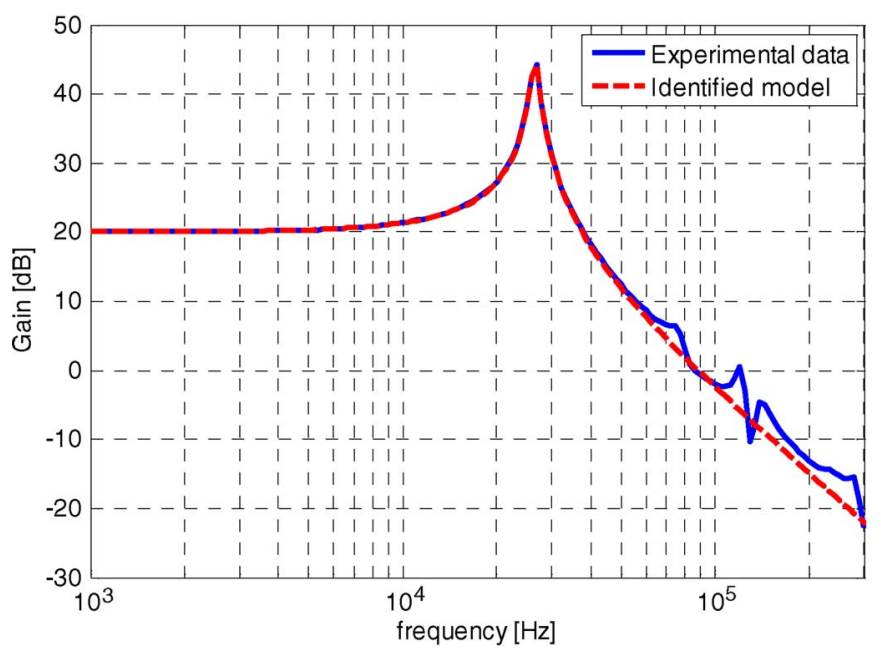

Fig. 12. Cross-validation of parameter identification based on $n_{2}$ testidentified model on $n_{2}$ compared with test $n_{1}\left(V_{2} / V_{1}\right)$.

$100 \mathrm{kHz}$ on the experimental data in Fig. 11 and difference appearing beyond $700 \mathrm{kHz}$. These differences are mainly due to two issues. The first one is given by the connection via $2-\mathrm{m}$ cable between the $H F$ amplifier and the transformer primarywinding terminals.

Indeed, the inductance of these cables is resonating with all the stray capacitances of the primary and secondary windings.

This has been verified owing to measurements with different cable lengths, leading to differences in the no-load impedance characteristic in frequencies beyond $700 \mathrm{kHz}$.
The second issue is related to the consideration of a model based on lumped parameters. To be more precise, each of the windings should be modeled with a higher order circuit in order to approach an exact solution; however, as previously mentioned, the designer necessitates the circuit in Fig. 3 for design model validation purposes with FEA, which are based on lumped parameters. The resonances appearing in the nearby of $100 \mathrm{kHz}$ are due to this model simplification.

However, to accurately represent the main performances of this transformer in normal operation, the model must be accurate up to a frequency of $\sim 40 \mathrm{kHz}$ (to represent a rise time on the order of $100 \mu \mathrm{s}$ and the converter's switching harmonic attenuation on the order of $10-20 \mathrm{kHz}$ ). It is clear that, for analyzing fast transients, such as arc events in the load (which may occur in klystrons), an increased-order model would be required.

\section{DISCUSSION}

Result interpretation of a physical model parametric identification can be very tedious. When the nonlinear optimization routine properly converges to a solution where the associated residuals tend to zero or to a white noise (from which no more information can be collected for identifying a deterministic model), one tends to conclude that the identification process is successful. It is true that, if the residuals are low enough, as in Figs. 11 and 12, the mathematical model represents the global system in an accurate way, which means that it can be used for high-performance control purposes. However, this is not enough to argue that the model is good enough for describing local physical behaviors, such as magnetic and electric fields' energy evolutions, represented by the parameters (need of the designer). Therefore, how can we evaluate the model accuracy in terms of its physical description? A good approach consists in cross-validating the estimated model on a different test, involving different inputs/outputs, which correspond to different state variables (as performed in Fig. 12), or considering different tests during the identification process. This is why it is very interesting to perform an analysis of the test selection. For instance, results of the sensitivity analysis in Table II on different tests lead to the same minimization of the residuals (no clear global minima in the objective function); however, the estimated parameters are not accurate with respect to the reference model. In other words, the parameters do not represent the local physical behavior of the system. In these cases, e.g., tests $n_{1}, n_{4}$, and $n_{5}$ in Table II, the models accurately represent the global behavior (input to output) of the specific test; however, a cross-validation on a different test would reveal that the model is actually heavily wrong. Therefore, cross-validating the estimated model, considering more than one test for the identification objective function determination, and performing a sensitivity analysis for the identification test selection (as in Section III) are all good ways of improving or testing the accuracy of the physical behavior of the estimated model.

During parametric identification, the consequences of neglecting nonlinear phenomena, such as magnetic saturation and skin effects, seem to be more important than reducing the model order with winding lumped parameters. Fig. 11 provides a 
very good illustration of this, considering that Fig. 12 suggests that the model order is sufficient enough. For faster pulse transformers (in the microsecond range of rise time), the model order would probably need to be increased.

\section{CONCLUSION}

The methodology for deriving transfer function models, for $H V$ pulse transformer nonlinear identification purposes, has been presented. The no-load primary impedance versus frequency test is the most adapted one for parametric identification purposes (at least for the megawatt-100-kV-range transformers). The importance of taking into account magnetic saturation and skin effects is very high and has been experimentally demonstrated with ML identification. Very good agreements between experimental and numerical results show the excellent efficiency of the proposed method.

\section{APPENDIX}

Coefficients of transfer functions corresponding to the two tests $\left(n_{1}\right.$ and $\left.n_{2}\right)$. Mathematical forms for MatLab "*.m" file. R2p corresponds to $R_{2}^{\prime}$, and L2p corresponds to $L_{2}^{\prime}$ in Fig. 3 .

For $n_{1}\left(V_{2} / V_{1}\right)$,

$$
\begin{aligned}
& \mathbf{A 1}=C 12 * L 1 * L 2 p * L m \\
& \mathbf{B 1}=C 12 *(L 1 * L m *(R 2 p+R f e) \\
& +L 2 p *(L 1 * R f e+L m *(R 1+R f e))) \\
& \mathbf{C 1}=C 12 *((L 2 p * R 1+L 1 * R 2 p) * R f e \\
& +L m *(R 2 p * R f e+R 1 *(R 2 p+R f e))) \\
& \mathbf{D 1}=(L m+C 12 * R 1 * R 2 p) * R f e \\
& \mathbf{E} 1=(C 12+C 2 p) * L 1 * L 2 p * L m \\
& \mathbf{F 1}=(C 12+C 2 p) *(L 1 * L m *(R 2 p+R f e)+L 2 p * \\
& \times(L 1 * R f e+L m *(R 1+R f e))) \\
& \mathbf{G} \mathbf{1}=L 1 *(L m+(C 12+C 2 p) * R 2 p * R f e) \\
& +(C 12+C 2 p) *(L 2 p * R 1 * R f e \\
& +L m *(R 2 p * R f e \\
& +R 1 *(R 2 p+R f e))) \\
& \mathbf{H 1}=(L 1+(C 12+C 2 p) * R 1 * R 2 p) * R f e \\
& +L m *(R 1+R f e) \\
& \mathbf{I} 1=R 1 * R f \text {. }
\end{aligned}
$$

For $n_{2}\left(V_{1} / I_{1}\right.$ at no load $)$,

$$
\begin{aligned}
& \mathbf{A 2}=(C 12+C 2 p) * L 1 * L 2 p * L m \\
& \mathbf{B 2}=(C 12+C 2 p) *(L 1 * L m *(R 2 p+R f e) \\
& +L 2 p *(L 1 * R f e+L m *(R 1+R f e))) \\
& \mathbf{C 2}=L 1 *(L m+(C 12+C 2 p) * R 2 p * R f e) \\
& +(C 12+C 2 p) *(L 2 p * R 1 * R f e+L m \\
& *(R 2 p * R f e+R 1 *(R 2 p+R f e)))
\end{aligned}
$$

$$
\begin{aligned}
& \mathbf{D 2}=(L 1+(C 12+C 2 p) * R 1 * R 2 p) * R f e \\
& +L m *(R 1+R f e) \\
& \mathbf{E 2}=R 1 * R f e \\
& \mathbf{F 2}=(C 12 * C 2 p+C 1 *(C 12+C 2 p)) \\
& \text { * } L 1 * L 2 p * L m \\
& \mathbf{G 2}=(C 12 * C 2 p+C 1 *(C 12+C 2 p)) \\
& \text { * }(L 1 * L m *(R 2 p+R f e) \\
& +L 2 p *(L 1 * R f e+L m *(R 1+R f e))) \\
& \mathbf{H 2}=C 1 * L 1 * L m+C 2 p * L 2 p * L m \\
& +C 12 *(L 1+L 2 p) * L m \\
& +C 12 * C 2 p * L m * R 1 * R 2 p \\
& +C 1 *(C 12+C 2 p) * L m * R 1 * R 2 p \\
& +(C 12 * C 2 p+C 1 *(C 12+C 2 p)) \\
& *((L 2 p+L m) * R 1+(L 1+L m) * R 2 p) \\
& \text { * Rfe } \\
& \mathbf{I 2}=C 1 * L m * R 1+C 12 * L m * R 1 \\
& +C 12 * L m * R 2 p+C 2 p * L m * R 2 p \\
& +(C 2 p *(L 2 p+L m) \\
& +C 12 *(L 1+L 2 p+C 2 p * R 1 * R 2 p) \\
& +C 1 *(L 1+L m+(C 12+C 2 p) * R 1 * R 2 p)) \\
& * R f e \\
& \mathbf{L} \mathbf{2}=L m+((C 1+C 12) * R 1+(C 12+C 2 p) * R 2 p) \\
& \text { * Rfe } \\
& \mathbf{M 2}=R f e .
\end{aligned}
$$

\section{REFERENCES}

[1] E. J. N. Wilson, An Introduction to Particle Accelerators. New York, NY, USA: Oxford Univ. Press, Aug. 2001, p. 272.

[2] C. A. Martins, F. Bordry, and G. Simonet, "A solid state $100 \mathrm{kV}$ long pulse generator for klystrons power supply," in Proc. 13th EPE, 2009, pp. 1-10.

[3] M. Kempkes, K. Schrock, R. Ciprian, T. Hawkey, M. P. J. Gaudreau, and A. Letchford, "A klystron power system for the ISIS front end test stand," in Proc. IEEE PPC, Jun. 28-Jul. 2, 2009, pp. 1335-1338.

[4] G. E. Leyh, "The ILC Marx modulator development program at SLAC," in Proc. IEEE PPC, Jun. 2005, pp. 1025-1028.

[5] C. Burkhart, T. Beukers, M. Kemp, R. Larsen, K. Macken, M. Nguyen, J. Olsen, and T. Tang, "ILC Marx modulator development program status," in Proc. IEEE PPC, Jun. 28-Jul. 2, 2009, pp. 807-810.

[6] M. Blajan, A. Umeda, S. Muramatsu, and K. Shimizu, "Emission spectroscopy of pulsed powered microplasma for surface treatment of PEN film," IEEE Trans. Ind. Appl., vol. 47, no. 3, pp. 1100-1108, May/Jun. 2011.

[7] H. Pfeffer, L. Bartelson, K. Bourkland, C. Jensen, Q. Kerns, P. Prieto, G. Saewert, and D. Wolff, "A long pulse modulator for reduced size and cost," in Proc. 21 st Int. Power Modulator Symp., Jun. 1994, pp. 48-51.

[8] R. Wagner, A. Bruesewitz, S. Choroba, H. J. Eckoldt, F. Eints, T. Froelich, T. Grevsmuehl, J. Hartung, A. Hauberg, L. Jachmann, J. Kahl, W. Koehler, H. Leich, N. Ngada, M. Penno, N. Poggensee, I. Sandvoss, I. Sokolov, V. Vogel, and R. Wenndorff, "The bounder modulators at DESY," in Proc. IET Eur. Pulsed Power Conf., Sep. 2009, pp. 1-4.

[9] D. Bortis, J. Biela, and J. W. Kolar, "Transient behavior of solid-state modulators with matrix transformers," IEEE Trans. Plasma Sci., vol. 38, no. 10 , pp. 2785-2792, Oct. 2010.

[10] D. Aguglia, C. A. Martins, M. Cerqueira Bastos, D. Nisbet, D. Siemaszko, E. Sklavounou, and P. Viarouge, "Klystron modulator technology 
challenges for the compact linear collider (CLIC)," in Proc. IEEE Conf. Pulsed Power, Washington, DC, USA, 2011, pp. 1413-1421.

[11] D. Aguglia, "2 MW Active bouncer converter design for long pulse klystron modulators," in Proc. 14th EPE, 2011, pp. 1-10.

[12] S. Maestri, R. G. Retegui, P. Antoszczuk, M. Benedetti, D. Aguglia, and D. Nisbet, "Improved control strategy for active bouncers used in klystron modulators," in Proc. 14th EPE, 2011, pp. 1-7.

[13] J. Bak-Jensen, B. Bak-Jensen, S. D. Mikkelsen, and C. G. Jensen, "Parametric identification in potential transformer modeling," IEEE Trans. Power Del., vol. 7, no. 1, pp. 70-76, Jan. 1992.

[14] J. Welsh, C. R. Rojas, and S. D. Mitchell, "Wideband parametric identification of a power transformer," in Proc. AUPEC, 2007, pp. 1-6.

[15] P. L. Mao, Z. Q. Bo, R. K. Aggarwal, and R. M. Li, "Identification of electromagnetic transients in power transformer system using artificial neural network," in Proc. Int. Conf. Power Syst. Technol., 1998, pp. 880-884.

[16] G. M. V. Zambrano, A. C. Ferreira, and L. P. Cablôba, "Power transformer equivalent circuit identification by artificial neural network using frequency response analysis," in Proc. IEEE Power Eng. Soc. Gen. Meet., 2006, pp. 1-6.

[17] P. Viarouge, D. Aguglia, C. A. Martins, and J. Cros, "Modeling and dimensioning of high voltage pulse transformers for klystron modulators," in Proc. ICEM, 2012, pp. 2332-2338.

[18] IEEE Standard for Pulse Transformers, ANSI/IEEE Std. 390-1987, 1987.

[19] H. Akcay, S. M. Islam, and B. Ninness, "Subspace-based identification of power transformer models from frequency response data," IEEE Trans. Instrum. Meas., vol. 48, no. 3, pp. 700-704, Jun. 1999.

[20] A. E. Kennelly, "Equivalence of triangles and stars in conducting networks," Elect. World Eng., vol. 34, pp. 413-414, Sep. 1899.

[21] J. D. Greene and C. A. Gross, "Nonlinear modeling of transformers," IEEE Trans. Ind. Appl., vol. 24, no. 3, pp. 434-438, May/Jun. 1988.

[22] A. Boglietti, A. Cavagnino, and M. Lazzari, "Experimental highfrequency parameters identification of AC electrical motors," IEEE Trans. Ind. Appl., vol. 43, no. 1, pp. 23-29, Jan./Feb. 2007.

[23] I. Kamwa, P. Viarouge, H. Le-Huy, and J. Dickinson, "A frequencydomain maximum likelihood estimation of synchronous machine highorder models using SSFR test data," IEEE Trans. Energy Convers., vol. 7, no. 3, pp. 525-535, Sep. 1992.

[24] T. C. Monteiro, F. O. Martinz, L. Matakas, and W. Komatsu, "Transformer operation at deep saturation: Model and parameter determination," IEEE Trans. Ind. Appl., vol. 48, no. 3, pp. 1054-1063, May/Jun. 2012.

[25] P. C. Krause, O. Wasynczuk, and S. D. Sudhoff, Analysis of Electric Machinery and Drive Systems, 2nd ed. Hoboken, NJ, USA: Wiley, 2002, p. 613.

[26] T. Tuovinen, M. Hinkkanen, and J. Luomi, "Modeling of saturation due to main and leakage flux interaction in induction machines," IEEE Trans. Ind. Appl., vol. 46, no. 3, pp. 937-945, May/Jun. 2010.

[27] R. Wamkeue, D. Aguglia, M. Lakehal, and P. Viarouge, "Two-step method for identification of nonlinear model of induction machine," IEEE Trans. Energy Convers., vol. 22, no. 4, pp. 801-809, Dec. 2007.

[28] O. M. O. Gatous and J. Pissolato, "Frequency-dependent skin-effect formulation for resistance and internal inductance of a solid cylindrical conductor," IET J. Microw., Antennas Propag., vol. 151, no. 3, pp. 212-216, Jun. 2004.

[29] J. Chatelain, Machines Electriques_-Volume X, Traité d'Électricité Series. Laussanne, Switzerland: Presses Polytechniques Romandes, 1983, p. 630, Éditions Georgi.

[30] H. A. Wheeler, "Skin resistance of a transmission-line conductor of polygon cross section," Proc. IRE, vol. 43, no. 7, pp. 805-808, Jul. 1955.

[31] A. Keyhani, S. Hao, and R. P. Schulz, "Maximum likelihood estimation of generator stability constants using SSFR test data," IEEE Trans. Energy Convers., vol. 6, no. 1, pp. 140-154, Mar. 1991

[32] R. Wamkeue, I. Kamwa, X. Dai-Do, and A. Keyhani, "Iteratively reweighted least squares for maximum likelihood identification of synchronous machine parameters from on-line tests," IEEE Trans. Energy Convers., vol. 14, no. 2, pp. 159-166, Jun. 1999.

[33] S. Tumageanian, A. Keyani, S. Moon, T. I. Leksan, and L. Xu, "Maximum likelihood estimation of synchronous machine parameters from flux decay data," IEEE Trans. Ind. Appl., vol. 30, no. 2, pp. 433-439, Mar./Apr. 1994.

[34] S. M. Islam, K. M. Coates, and G. Ledwich, "Identification of high frequency transformer equivalent circuit using Matlab from frequency domain data," in Conf. Rec. IEEE IAS Annu. Meeting, New Orleans, LA, USA, 1997, pp. 357-364

[35] R. E. Maine and K. W. Iliff, "Formulation and implementation of a practical algorithm for parameter estimation with process and measurement noise," SIAM J. Appl. Math., vol. 41, no. 3, pp. 558-579, Dec. 1981.

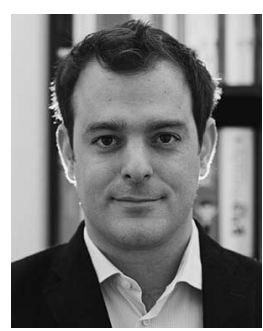

Davide Aguglia (S'06-M'08) was born in Switzerland in 1979. He received the Electrical Engineering Diploma from the Ecole d'Ingénieurs et d'Architectes de Fribourg, University of Applied Science of Western Switzerland, Fribourg, Switzerland, in 2002 and the M.Sc. and Ph.D. degrees in electrical engineering from Laval University, Québec City, QC, Canada, in 2004 and 2010, respectively.

Since 2008, he has been with the Power Converter Group, Technology Department, European Organization for Nuclear Research (CERN), Geneva, Switzerland, where he is responsible for fast pulsed converter design and commissioning. He has been an External Consultant for a private company in magnetic component design, and since January 2011, he has also been an Associate Professor with Laval University, where he is codirecting Ph.D. students. He is leading the international $\mathrm{R} \& \mathrm{D}$ program on klystron modulator design for the next generation of particle accelerators (Compact Linear Collider Studies). His main research interests are power electronics system design and high-voltage engineering for particle accelerators, as well as electrical machines and renewable energy system design.

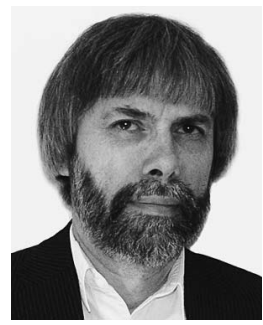

Philippe Viarouge (M'93) was born in Périgueux, France, in 1954. He received the Engineering and Doctor of Engineering degrees and the French Accreditation to Supervise Research (HDR) from the Institut National Polytechnique de Toulouse, Toulouse, France, in 1976, 1979, and 1992, respectively.

In 2009-2010, he was a Project Associate with the European Organization for Nuclear Research (CERN), Geneva, Switzerland. Since 1979, he has been a Professor with the Department of Electrical Engineering, Laval University, Quebec City, QC, Canada, where he is currently working in the research laboratory "Laboratoire d'Électrotechnique, Électronique de Puissance et Commande Industrielle." His research interests include the design and modeling of electrical machines and medium-frequency magnetic components, ac drives, and power electronics.

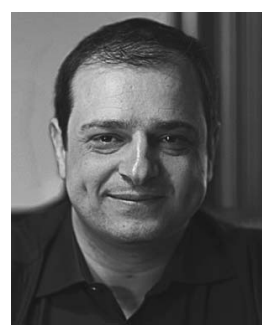

Carlos de Almeida Martins (S'93-M'01) was born in Portugal in 1973. He received the B.Eng. degree in electrical and computer engineering from the University of Porto, Porto, Portugal, in 1996 and the M.Sc. and Ph.D. degrees in electrical engineering from the Institut National Polytechnique de Toulouse, Toulouse, France, in 1997 and 2000, respectively, in the field of power electronics.

From 2001 to 2009, he was a Staff Member with the European Organization for Nuclear Research (CERN), where he worked as an R\&D Engineer in the Power Converters Group with several responsibilities in the design, development, specification, testing, and commissioning of different types of high-power supplies for the CERN accelerator complex. He was appointed as a Section Leader in the "High Voltage and Pulsed Power Converters" Section in 2006. From April 2011, he was a Professor with the Department of Electrical Engineering, Laval University, Québec City, QC, Canada, where he was a Researcher in the Laboratoire d'Électrotechnique, Électronique de Puissance et Commande Industrielle. Since January 2013, he has been with the European Spallation Source, Lund, Sweden. His current research interests include highpower electronics for transport and distribution applications and pulsed-power electronics for physics and medical applications. 